\title{
The effect of COVID-19 positivity on inflammatory parameters and thirty day mortality rates in patients over sixty five years of age with surgically treated intertrochanteric fractures
}

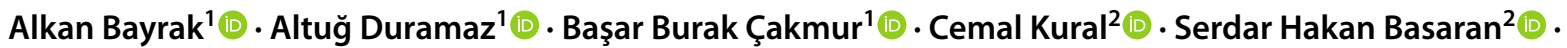

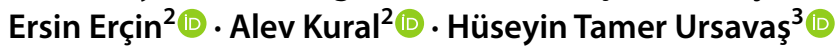

Received: 15 June 2021 / Accepted: 22 August 2021 / Published online: 1 September 2021

(c) SICOT aisbl 2021

\begin{abstract}
Purpose To evaluate the effect of COVID-19 positivity on inflammatory parameters and 30-day mortality rates in patients over 65 years of age who were operated on for intertrochanteric femur fractures (IFF).

Methods Eighty-seven patients (31 males, 56 females) who had a dynamic hip screw (DHS) or proximal femur nail (PFN) for the IFF between March 2020 and November 2020 were included in the study. The patients were divided into two groups as COVID-19 confirmed and probable positive (Group 1) and COVID-19 negative (Group 2). Time to surgery, operation duration, length of hospital stay, 30-day mortality, rates of the intensive care unit (ICU) referral, and inflammatory parameters such as haemoglobin, CRP, sedimentation, PCT, D-Dimer, and ferritin were evaluated.

Results No significant difference was observed in terms of demographic data such as age, gender, comorbidity, and fracture type between the groups. Thirty-day mortality, ICU referral rate, blood transfusion rate, and hospitalization period were higher in Group 1 ( $p=0.016, p=0.012, p=0.031$, and $p=0.011$, respectively). The inflammatory parameters were higher in Group 1 compared to Group 2 in the preoperative and postoperative periods $(p<0.05)$.

Conclusion COVID-19 positivity increases inflammatory parameters (as expected) and increases the 30-day mortality and ICU requirement in patients with surgically treated IFF.
\end{abstract}

Keywords COVID-19 $\cdot$ Intertrochanteric femur fracture $\cdot$ Inflammation $\cdot$ Mortality

Alkan Bayrak

drqueum@gmail.com

Altuğ Duramaz

altug.duramaz@yahoo.com

Başar Burak Çakmur

basar_cakmur@hotmail.com

Cemal Kural

cemalkural@hotmail.com

Serdar Hakan Basaran

drserdarhakan@gmail.com

Ersin Erçin

ersine@ hotmail.com

Alev Kural

alevkural@hotmail.com
Hüseyin Tamer Ursavaş

htamerursavas@gmail.com

1 Department of Orthopedics and Traumatology, Bakırköy Dr. Sadi Konuk Training and Research Hospital, Tevfik Sağlam St. Number 11, 34147 Bakırköy, Istanbul, Turkey

2 Department of Orthopedics and Traumatology, University of Health Sciences, Bakırköy Dr. Sadi Konuk Training and Research Hospital, Tevfik Sağlam St. Number 11, 34147 Bakırköy, Istanbul, Turkey

3 Orthopedics and Traumatology, Silifke State Hospital, Koyuncu Sok. No:434, 33960 Mersin, Turkey 


\section{Introduction}

The pandemic caused by the COVID-19 (SARS Cov-2 virus) was first seen in the Hubei province of China in 2019 [1]. Various measures have been taken in our country to control the effects of the epidemic after the cases started to appear in March 2020 [2]. Measures such as limiting elective orthopaedic surgery and restricting people over 65 and under 20 from going out on the streets were among the measures taken in this context [2]. Although people over the age of 65 were restricted from going out to the streets, intertrochanteric femur fractures (IFFs) frequently continued in elderly patients due to low-energy trauma such as simple falls. IFFs are an important factor affecting mortality and morbidity in this patient group because elderly patients are often accompanied by comorbidities [3]. While IFFs were treated during the pandemic, COVID-19 positive patients were operated on in negative-pressure operating rooms and the surgical team entered surgery with personal protective equipment $[4,5]$. Clinical symptoms, PCR test from oral and nasopharyngeal swabs, procalcitonin (PCT), C-reactive protein (CRP), sedimentation (ESR), lactate dehydrogenase (LDH), D-Dimer, ferritin, and imaging methods (chest radiography and thoracic computed tomography) have been used for the diagnosis of COVID-19 and patient follow-up [1, 6]. Although the relationship between biochemical values and disease progression is controversial in the literature, its clinical use continues [7, 8].

The systemic immune inflammation index (SII) as a systemic inflammation marker has been used as a prognostic factor in some cancer types and inflammatory diseases in recent years $[9,10]$. SII index is calculated by multiplying the platelet count by the neutrophil count and dividing by the lymphocyte count [10]. Inflammation is also known to increase in COVID-19 disease [1]. In our study, it was evaluated whether the SII index was different between COVID-19 positive and negative patient groups.

The present study is aimed to evaluate the effect of COVID-19 positivity on inflammatory parameters, ICU referral rate, and 30-day mortality rates in patients over 65 years of age who underwent surgery for IFF. We hypothesized that COVID-19 positive patients would have a higher hospital stay, time to surgery, mortality, and referral rates for ICU, and inflammatory parameters.

\section{Material and methods}

In this retrospective study, patients over the age of 65 who underwent surgery for IFF between April 2020 and November 2020 were evaluated after the approval of the ethics committee (Ethical committee ID: AB-2020-0622T19 0336 and 2020/326). Patients who underwent dynamic hip nail (DHS) and proximal femoral nail (PFN) due to IFF were included in the study. Based on real-time reverse transcriptase-polymerase chain reaction (PCR) positivity, clinical symptoms, chest radiographs, and chest computed tomography (CT) patients were stratified into COVID-19 positive and COVID-19 negative subgroups. Suspected COVID-19 patients (PCR negative, + clinical symptoms, + CT) were accepted as COVID-19 positive. AO/ OTA classification was used in the fracture classification. Partial hip arthroplasty, total hip arthroplasities, proximal femoral plate fixation, pathological fractures, patients who underwent additional surgical treatment other than hip surgery, revision surgery, and patients under 65 years of age were excluded from the study. The demographic data of the patients, time to operation, operation duration, hospitalization period, the need for blood transfusion, mortality, and referral rates to ICU were evaluated. PCR test, chest imaging, and blood analysis were performed in all patients scheduled for surgery to identify COVID-19 positive patients during the pandemic period. Chest radiography was evaluated routinely. CT was evaluated in addition to chest radiography in symptomatic patients with symptoms such as high fever, cough, myalgia, and loss of taste and smell. Inflammatory parameters such as PCT, CRP, ESR, LDH, D-Dimer, and ferritin, as well as preoperative and postoperative haemoglobin, hematocrit, neutrophil, lymphocyte, platelet, sodium, potassium, PT, aPTT, INR, urea, creatinine, were evaluated. This was routinely applied both for follow-up of patients and to protect healthcare providers against false PCR negativity. Treatment protocols (antiviral drugs, hydroxychloroquine, enoxaparin), which were determined by the Ministry of Health and renewed according to scientific data, were started for confirmed and probable COVID-19 positive patients, and the treatment of the patients was planned and surgical preparations were made by taking them to an isolated service [11-13]. COVID-19 negative patients are followed up in the orthopaedic service under normal conditions. $94.25 \%(n=82)$ of the patients were operated under spinal anesthesia, $5.75 \%(n=5)$ under general anesthesia. The preoperative and postoperative laboratory examinations of the patients were evaluated and checked at 48-72-hour intervals according to the patients' clinic and for the followup of the treatment. The patients were encouraged to partial weight-bearing in the postoperative period.

\section{Statistical analysis}

NCSS (Number Cruncher Statistical System) 2007 (Kaysville, UT, USA) program was used for statistical analysis. Descriptive statistical methods (median, first quarter, 
third quarter) were used when evaluating the study data. The suitability of quantitative data for normal distribution was tested by the Shapiro-Wilk test and graphical examinations. Student's $t$-test was used to compare normally distributed quantitative variables between two groups and Mann-Whitney's $U$ test was used to compare the quantitative variables that did not show normal distribution between the two groups. Dependent groups $t$-test was used for in-group comparisons of quantitative variables with normal distribution. Wilcoxon's signed-ranks test was used for intragroup comparisons of quantitative variables that did not show normal distribution. Pearson's chi-square test, Fisher's exact test, and Fisher-Freeman-Halton's test were used to compare qualitative data. Statistical significance was accepted as $p<0.05$.

\section{Results}

The demographic features of the patients are presented in Table 1 . No statistically significant difference was found between COVID-19 positive patients and COVID-19 negative patients between age, gender, time to surgery, operation duration, postoperative femoral neck-shaft angle, SII index,
Table 1 Demographic features of patients

\begin{tabular}{|c|c|c|c|c|}
\hline & & COVID (-) & COVID (+) & $p$ \\
\hline Age (year) & $M e a n \pm S D$ & $79.0 \pm 12.4$ & $79.5 \pm 14.9$ & ${ }^{a} 0.863$ \\
\hline \multirow[t]{2}{*}{ Gender } & Male & $22(34.9)$ & $9(37.5)$ & \multirow[t]{2}{*}{${ }^{b} 0.822$} \\
\hline & Female & $41(65.1)$ & $15(62.5)$ & \\
\hline Operation duration (min) & $O r t \pm S s$ & $64.0 \pm 9.7$ & $67.5 \pm 11.1$ & ${ }^{a} 0.157$ \\
\hline Time to surgery (hours) & Median $(Q 1-Q 3)$ & $28(24-36)$ & $34(31-52)$ & ${ }^{c} 0.479$ \\
\hline Hospitalization period (day) & Median $(Q 1-Q 3)$ & $9(7-11)$ & $11(8.5-15.5)$ & ${ }^{c} 0.011 *$ \\
\hline Femur head-neck angle & $M e a n \pm S D$ & $11.3 \pm 1.7$ & $11.6 \pm 2.1$ & ${ }^{a} 0.523$ \\
\hline SII index & Mean $\pm S D$ & $\begin{array}{l}1728.8+1195.8 \\
\boldsymbol{n}(\%)\end{array}$ & $\begin{array}{l}1578.5+765.1 \\
\boldsymbol{n}(\%)\end{array}$ & 0.45 \\
\hline \multirow[t]{3}{*}{ ASA } & I & $1(1.6)$ & $0(0)$ & \multirow[t]{3}{*}{${ }^{d} 0.688$} \\
\hline & II & $16(25.4)$ & $4(16.7)$ & \\
\hline & III & $46(73)$ & $20(83.3)$ & \\
\hline \multirow[t]{9}{*}{ Fracture type } & A01.1 & $3(4.8)$ & $0(0)$ & \multirow[t]{9}{*}{${ }^{d} 0.063$} \\
\hline & A01.2 & $7(11.1)$ & $1(4.2)$ & \\
\hline & A01.3 & $2(3.2)$ & $0(0)$ & \\
\hline & A02.1 & $16(25.4)$ & $5(20.8)$ & \\
\hline & A02.2 & $11(17.5)$ & $10(41.7)$ & \\
\hline & A02.3 & $12(19)$ & $2(8.3)$ & \\
\hline & A03.1 & $3(4.8)$ & $4(16.7)$ & \\
\hline & A03.3 & $9(14.3)$ & $1(4.2)$ & \\
\hline & $\mathrm{A} 2.2$ & $0(0)$ & $1(4.2)$ & \\
\hline \multirow[t]{2}{*}{ PCR } & Negative & $63(100)$ & $11(45.8)$ & \multirow[t]{2}{*}{${ }^{b} 0.031 *$} \\
\hline & Positive & $0(0)$ & $13(54.2)$ & \\
\hline \multirow[t]{2}{*}{ Chest CT } & Negative & $63(100)$ & $2(8.3)$ & \multirow[t]{2}{*}{${ }^{b} 0.031 *$} \\
\hline & Positive & $0(0)$ & $22(91.7)$ & \\
\hline \multirow[t]{2}{*}{ Treatment } & No & $63(100)$ & $4(16.7)$ & \multirow[t]{2}{*}{${ }^{b} 0.031^{*}$} \\
\hline & Yes & $0(0)$ & $20(83.3)$ & \\
\hline Comorbidity & No & $20(31.7)$ & $6(25)$ & ${ }^{b} 0.460$ \\
\hline Hipertension & & $15(23.8)$ & $5(20.8)$ & \\
\hline Diabetes mellitus & & $19(30.2)$ & $6(25)$ & \\
\hline $\begin{array}{l}\text { Others (stroke, chronic renal } \\
\text { failure, etc.) }\end{array}$ & & $9(14.3)$ & $7(29.2)$ & \\
\hline \multicolumn{5}{|l|}{ aStudent's $t$ test } \\
\hline \multicolumn{5}{|l|}{${ }^{\text {b}}$ Pearson's chi-square test } \\
\hline \multicolumn{5}{|l|}{ 'Mann-Whitney's $U$ test } \\
\hline \multicolumn{5}{|l|}{${ }^{\mathrm{d}}$ Fisher-Freeman-Halton's test } \\
\hline${ }^{*} p<0.05$ & & & & \\
\hline
\end{tabular}


ASA score, fracture type, and comorbidities $(p>0.05)$. The hospitalization period, need for blood transfusion, referral to ICU, and the mortality rate was found to be statistically high in COVID-19 positive patients $(p=0.011, p=0.031$, $p=0.012$, and $p=0.016$, respectively) (Tables 1 and 2). Preoperative and postoperative haemoglobin measurements and changes in measurements according to COVID19 positivity do not show a statistically significant difference $(p>0.05)$. Postoperative haemoglobin decrease was found to be statistically significant in both COVID-19 negative and COVID-19 positive patients compared to preoperative values $(p=0.001)$. No statistically significant difference was found between the preoperative neutrophil to lymphocyte rate (NLR) of the patients according to COVID-19 positivity $(p>0.05)$. The postoperative NLR of COVID-19 negative patients was statistically significantly lower than the COVID-19 positive patients $(p=0.010)$. The decrease in postoperative NLR in COVID-19 positive patients compared to preoperative NLR was found to be statistically significant $(p=0.002)$. The change in postoperative NLR in COVID-19 positive patients compared to preoperative was statistically significantly lower than COVID-19 negative patients $(p=0.007)$. Preoperative and postoperative LDH values of COVID-19 negative patients were statistically significantly lower than COVID-19 positive patients ( $p=0.009$ and $p=0.011$, respectively). The preoperative and postoperative AST to ALT ratios of the patients do not show a statistically significant difference in terms of COVID-19 positivity $(p>0.05)$. The increase in postoperative AST/ALT ratio in COVID-19 negative patients and COVID-19 positive patients compared to preoperative AST/ ALT ratio was found to be statistically significant $(p=0.001$ and $p=0.013$, respectively). The postoperative D-dimer of COVID-19 negative patients was statistically significantly lower than COVID-19 positive patients $(p=0.005)$. The decrease in postoperative D-dimer of COVID-19 negative

Table 2 Evaluation of mortality rate, ICU referral rate, and blood transfusion rate between the COVID-19 positive and COVID-19 negative patients

\begin{tabular}{lllll}
\hline & & $\begin{array}{l}\text { COVID }(-) \\
n(\%)\end{array}$ & $\begin{array}{l}\text { COVID }(+) \\
n(\%)\end{array}$ & $p$ \\
\hline Blood transfusion & No & $29(46.0)$ & $5(20.8)$ & ${ }^{b} 0.031^{*}$ \\
& Yes & $34(54.0)$ & $19(79.2)$ & \\
ICU referral & No & $60(95.2)$ & $18(75)$ & ${ }^{g} 0.012^{*}$ \\
& Yes & $3(4.8)$ & $6(25)$ & \\
Exitus & No & $61(96.8)$ & $19(79.2)$ & ${ }^{g} 0.016^{*}$ \\
& Yes & $2(3.2)$ & $5(20.8)$ & \\
\hline
\end{tabular}

${ }^{\mathrm{b}}$ Pearson's chi-square test

gFisher's exact test

${ }^{*} p<0.05, * * p<0.01$ patients compared to preoperative D-dimer was found to be statistically significant $(p=0.015)$. The change in postoperative D-dimer of COVID-19 negative patients compared to preoperative $\mathrm{D}$-dimer was statistically significantly lower than COVID-19 positive patients $(p=0.007)$. The increase in postoperative total protein to albumin rate in COVID19 negative and COVID-19 positive patients compared to preoperative total protein to albumin rate was found to be statistically significant ( $p=0.045$ and $p=0.001$ ). The postoperative CRP, ESR, PCT, and ferritin values of COVID-19 negative patients were statistically significantly lower than COVID-19 positive patients ( $p=0.001, p=0.001, p=0.035$, and $p=0.011$, respectively). The increase in postoperative CRP, ESR, PCT, and ferritin values of COVID-19 negative and COVID-19 positive patients compared to preoperative values was found to be statistically significant $(p=0.001$; $p=0.001, p=0.023$, and $p=0.001$, respectively). The change in post-op CRP, ESR, and ferritin values of COVID19 negative patients compared to preoperative values was statistically significantly lower than COVID-19 positive patients ( $p=0.001, p=0.007$, and $p=0.001$, respectively). The increase in postoperative PCT value of COVID-19 negative patients compared to preoperative values was found to be statistically significant $(p=0.035)$ (Table 3$)$.

\section{Discussion}

The presented study evaluated the demographic characteristics, duration of hospitalization, blood transfusion rates, mortality, morbidity, and inflammatory parameters in blood values of COVID-19 positive patients over 65 years of age who underwent surgery due to IFF during the pandemic period, and compared with COVID-19 negative patients. Patients over 65 years of age who suffer from hip fractures often have additional diseases and hip fracture affects mortality and morbidity in those patients [14]. COVID-19 also increases mortality and morbidity in this patient group, as it adversely affects the respiratory system [15]. Although it is stated that the postoperative 30 -day mortality rate is $7-15 \%$ in the COVID-19 negative population, this rate reaches up to $40 \%$ in the COVID-19 positive patients $[16,17]$. In our study, $20.8 \%$ of COVID-19 positive patients died, while $25 \%$ were referred to ICU. While $3.2 \%$ of COVID-19 negative patients died, $4.8 \%$ were referred to the ICU. The COVID- 19 positive patients' ex rate was found to be more than six times higher than the COVID-19 negative patients.

The literature shows that COVID-19 positive patients have longer surgical and hospital stays compared to COVID19 negative patients [18]. Management of COVID-19 positive patients and their need for additional treatments such as respiratory support in the postoperative period extend the hospitalization period $[19,20]$. In addition, the duration of 
Table 3 Evaluation of biochemical analysis between the COVID-19 positive and COVID-19 negative patients

\begin{tabular}{|c|c|c|c|c|}
\hline & & COVID (-) & COVID (+) & $p$ \\
\hline Preoperative $\mathrm{Hb}$ (gr/dl) & Mean $\pm S D$ & $11.3 \pm 1.7$ & $11.6 \pm 2.1$ & ${ }^{a} 0.461$ \\
\hline \multirow[t]{2}{*}{ Postoperative $\mathrm{Hb}$ (gr/dl) } & $M e a n \pm S D$ & $10.0 \pm 1.5$ & $9.7 \pm 1.6$ & ${ }^{a} 0.415$ \\
\hline & $p$ & ${ }^{e} \mathbf{0 . 0 0 1} * *$ & ${ }^{e} \mathbf{0 . 0 0 1 * *}$ & ${ }^{c} 0.131$ \\
\hline Preoperative Neutrophil/lymphocyte ratio (10e3/uL) & Median $(Q 1-Q 3)$ & $6.7(4.8-10.5)$ & $7.7(3-11.5)$ & ${ }^{c} 0.947$ \\
\hline \multirow[t]{2}{*}{ Postoperative Neutrophil/lymphocyte ratio (10e3/uL) } & Median $(Q 1-Q 3)$ & $8(4.5-10.7)$ & $11.6(7-17.1)$ & ${ }^{c} \mathbf{0 . 0 1 0} *$ \\
\hline & $p$ & $f_{\mathbf{0 . 5 2 7}}$ & $f_{\mathbf{0 . 0 0 2}} * *$ & ${ }^{c} \mathbf{0 . 0 0 7} * *$ \\
\hline Preoperative LDH (U/L) & Median $(Q 1-Q 3)$ & $223(161-269)$ & $290(207-352.5)$ & ${ }^{c} 0.009 * *$ \\
\hline \multirow[t]{2}{*}{ Postoperative LDH (U/L) } & Median $(Q 1-Q 3)$ & $215(165-282)$ & $258.5(205.5-338)$ & ${ }^{c} \mathbf{0 . 0 1 1} *$ \\
\hline & $p$ & $f_{\mathbf{0 . 3 3 1}}$ & $f_{\mathbf{0 . 7 4 2}}$ & ${ }^{c} 0.962$ \\
\hline Preoperative urea (mg/dl) & Median $(Q 1-Q 3)$ & $48(36-73)$ & $48(39-68.5)$ & ${ }^{c} 0.928$ \\
\hline \multirow[t]{2}{*}{ Postoperative urea (mg/dl) } & Median $(Q 1-Q 3)$ & $59(38-80)$ & $60(35-92.5)$ & ${ }^{c} 0.393$ \\
\hline & $p$ & $f_{\mathbf{0 . 0 3 8}} *$ & $f_{\mathbf{0 . 0 1 1}} *$ & ${ }^{c} 0.117$ \\
\hline Preoperative AST/ALT (U/L) & Mean $\pm S D$ & $1.8 \pm 0.6$ & $1.7 \pm 0.7$ & ${ }^{a} 0.577$ \\
\hline \multirow[t]{2}{*}{ Postoperative AST/ALT (U/L) } & Mean $\pm S D$ & $2.5 \pm 1.4$ & $2.5 \pm 1.7$ & ${ }^{a} 0.990$ \\
\hline & $p$ & ${ }^{e} 0.001 * *$ & ${ }^{e} 0.013 *$ & ${ }^{c} 0.966$ \\
\hline Preoperative LDL (mg/dl) & Median $(Q 1-Q 3)$ & $128(97-150)$ & $125(111-172)$ & ${ }^{c} 0.659$ \\
\hline \multirow[t]{2}{*}{ Postoperative LDL (mg/dl) } & Median $(Q 1-Q 3)$ & $124(92-150)$ & $125.5(88-172.5)$ & ${ }^{c} 0.905$ \\
\hline & $p$ & $f_{\mathbf{0 . 0 5 5}}$ & ${ }^{f} \mathbf{0 . 8 8 4}$ & ${ }^{c} 0.138$ \\
\hline Preoperative D-Dimer (ug/L) & Median $(Q 1-Q 3)$ & $1.2(0.5-2.5)$ & $2(1.2-3.4)$ & ${ }^{c} 0.062$ \\
\hline \multirow[t]{2}{*}{ Postoperative D-Dimer (ug/L) } & Median $(Q 1-Q 3)$ & $1.1(0.4-2.1)$ & $2.2(1.2-3.4)$ & ${ }^{c} 0.005 * *$ \\
\hline & $p$ & $f_{0.015}^{*}$ & $f_{0.932}$ & ${ }^{c} 0.052$ \\
\hline Preoperative T-Prot/Alb (g/L) & $M e a n \pm S D$ & $1.81 \pm 0.18$ & $1.80 \pm 0.19$ & ${ }^{a} 0.766$ \\
\hline \multirow[t]{2}{*}{ Postoperative T-Prot/Alb (g/L) } & Mean $\pm S D$ & $1.86 \pm 0.20$ & $1.94 \pm 0.17$ & ${ }^{a} 0.098$ \\
\hline & $p$ & ${ }^{e} \mathbf{0 . 0 4 5} *$ & ${ }^{e} \mathbf{0 . 0 0 1 * *}$ & ${ }^{c} 0.109$ \\
\hline Preoperative CRP (mg/dl) & Median $(Q 1-Q 3)$ & $9(3-36)$ & $6(2-56.5)$ & ${ }^{c} 0.853$ \\
\hline \multirow[t]{2}{*}{ Postoperative CRP (mg/dl) } & Median $(Q 1-Q 3)$ & $27(11-81)$ & $149.5(97-245.5)$ & ${ }^{c} \mathbf{0 . 0 0 1} * *$ \\
\hline & $p$ & $f_{\mathbf{0 . 0 0 1}} * *$ & $f_{\mathbf{0 . 0 0 1}} * *$ & ${ }^{c} \mathbf{0 . 0 0 1} * *$ \\
\hline Preoperative ESR (mm/h) & Median (Q1-Q3) & $11(6-14)$ & $24.5(15-28)$ & ${ }^{c} 0.001 * *$ \\
\hline \multirow[t]{2}{*}{ Postoperative ESR (mm/h) } & Median $(Q 1-Q 3)$ & $15(8-19)$ & $29.5(23-38)$ & ${ }^{c} \mathbf{0 . 0 0 1} * *$ \\
\hline & $p$ & $f_{\mathbf{0 . 0 0 1} * *}$ & $f_{\mathbf{0 . 0 0 1} * *}$ & ${ }^{c} \mathbf{0 . 0 0 7 * *}$ \\
\hline Preoperative PCT (ng/mL) & Median $(Q 1-Q 3)$ & $0.1(0.1-0.2)$ & $0.1(0.1-0.3)$ & ${ }^{c} 0.479$ \\
\hline \multirow[t]{2}{*}{ Postoperative PCT (ng/mL) } & Median $(Q 1-Q 3)$ & $0.1(0.1-0.3)$ & $0.2(0.1-0.5)$ & ${ }^{c} \mathbf{0 . 0 3 5}^{*}$ \\
\hline & $p$ & $f_{\mathbf{0 . 0 3 5}}^{*}$ & $f_{0.232}$ & ${ }^{c} 0.369$ \\
\hline Ferritin (pre-treatment) $\mu \mathrm{g} / \mathrm{L}$ & Median $(Q 1-Q 3)$ & 148 (89-195) & $161.5(63-265)$ & ${ }^{c} 0.462$ \\
\hline \multirow[t]{2}{*}{ Ferritin (post-treatment) $\mu \mathrm{g} / \mathrm{L}$} & Median $(Q 1-Q 3)$ & $170(90-200)$ & $277.5(132.5-361.5)$ & ${ }^{c} 0.011^{*}$ \\
\hline & $p$ & $f_{\mathbf{0 . 0 2 3}} *$ & $f_{\mathbf{0 . 0 0 1} * *}$ & ${ }^{c} \mathbf{0 . 0 0 1} * *$ \\
\hline
\end{tabular}

Abbreviations: $H b$ hemeglobin, $L D H$ lactate dehydrogenase, $A S T$ aspartateaminotransferase, $A L T$ alanine aminotransferase, $L D L$ low density lipoprotein

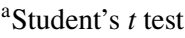

${ }^{\mathrm{c}}$ Mann-Whitney's $U$ test

${ }^{\text {e}}$ Paired samples test

${ }^{\mathrm{f}}$ Wilcoxon's signed-ranks test

${ }^{*} p<0.05, * * p<0.01$

the surgery is prolonged since COVID-19 positive patients are operated on in negative-pressure rooms in the theatre and by surgeons dressed with special equipment [18]. In our study, while the hospitalization periods of COVID-19 positive patients were found to be longer, the operation duration was found similar to COVID-19 negative patients. We think that the items below are shortened the duration of surgery (a) keeping COVID-19 positive and COVID-19 negative patients in separate departments, (b) performing all preparations and postoperative wake-up procedures in the same 
room when COVID-19 positive patients are taken into the operating room, (c) taking limited staff into the room and providing the necessary materials with additional medical personnel outside the operation room.

Several studies evaluating the parameters that correlate with the course of COVID-19 have shown that CRP, NLR, and ESR, which are frequently used as inflammation markers, increase in COVID-19 positive patients [6, 8]. In addition, ferritin, LDH, PCT, and D-Dimer also increase in the course of the disease in COVID-19 positive patients [21]. These parameters are also used in the clinical follow-up of the patients, to evaluate the progression of the disease and the response to treatment. The present study showed that PCT, ferritin, LDH, CRP, ESR, and NLR were higher in COVID-19 positive patients compared to COVID-19 negative patients. Although there was no statistically significant difference in haemoglobin values between COVID-19 positive patients and COVID-19 negative patients, it was observed that haemoglobin was low in the postoperative period in both patient groups. In COVID-19 positive patients, the need for blood transfusion was determined more than in COVID-19 negative patients. We think that more blood transfusions are performed because COVID19 positive patients are haemodynamically more unstable. In the literature, the severity of the disease in COVID-19 positive patients has been associated with various parameters such as age, comorbidities, CRP, ESR, neutrophil and lymphocyte count, PCT, and albumin [22, 23]. In the current study, it was observed that inflammation parameters were high in COVID-19 positive patients. Since elderly patients were evaluated in the present study, no difference was found between the COVID-19 positive and COVID19 negative patients in terms of age and comorbidity rates. COVID-19 positivity is not the only parameter that causes high CRP and increased NLR in COVID-19 patients, trauma and comorbidities are also affecting CRP and NLR [24]. Although COVID-19 inflammation with increased CRP and increased NLR is associated with higher ASA classification and higher comorbidity rate, no significant difference was observed between COVID-19 positive and negative patients in terms of ASA classification in the present study. Also, the SSI index was shown as a new marker in recent years and it correlates as a prognostic marker in some cancer types in the literature $[9,10]$. Because of the systemic inflammation SII index increases. In the current study, we did not consider any difference between groups. Although COVID19 provides a systemic inflammation, both groups have a traumatic hip fracture. Our opinion is due to this trauma SII index ratio similar in both groups. The limitations of our study are the small number of patients, short follow-up times, and retrospective design. However, the strength of our study is to examine patients over 65 years of age who were operated on for an isolated IFF. The present study is a pilot study evaluating 30-day mortality rates of the patients who underwent surgery for proximal femur fractures. As a result of long-term studies and studies with larger patient groups, it would be appropriate to evaluate the long-term effects of COVID-19 positivity.

In conclusion, urgent surgical treatment of elderly COVID-19 positive and COVID-19 negative patients and the use of inflammatory parameters in the course of COVID19 positive patients are important. It should be taken into account that COVID-19 positive patients may stay in the hospital for a longer period and the risk of 30-day mortality is higher.

Author contribution All the authors have approved the final contents of the submission and been actively involved in the planning and in the enactment of the study.

Data availability Not applicable.

Code availability Not applicable.

\section{Declarations}

Ethics approval All procedures performed in studies involving human participants were in accordance with the ethical standards of the institutional and/or national research committee and with the 1964 Helsinki declaration and its later amendments or comparable ethical standards. Bakırköy Dr. Sadi Konuk Training and Research Hospital Ethics Committee, Ethical Approval ID:2020/326. TR, Ministry of Health Study ID: AB-2020-06-22T19_03_36.

Consent to participate Not applicable.

Consent for publication Not applicable.

Conflict of interest The authors declare no competing interests.

\section{References}

1. Casiraghi A, Domenicucci M, Cattaneo S et al (2020) Operational strategies of a trauma hub in early coronavirus disease 2019 pandemic. Int Orthop 44(8):1511-1518. https://doi.org/10.1007/ s00264-020-04635-5

2. Carkci E, Polat B, Polat A, Peker B, Öztürkmen Y (2021) The Effect of the Coronavirus 2019 (COVID-19) pandemic on the number and characteristics of orthopedic trauma patients in a tertiary care hospital in Istanbul. Cureus 13(1):e12569. https://doi. org/10.7759/cureus. 12569

3. Wolf O, Mukka S, Ekelund J, Möller M, Hailer NP (2021) How deadly is a fracture distal to the hip in the elderly? An observational cohort study of 11,799 femoral fractures in the Swedish Fracture Register. Acta Orthop 92(1):40-46. https://doi.org/10. 1080/17453674.2020.1831236

4. LeBrun DG, Konnaris MA, Ghahramani GC et al (2020) Hip fracture outcomes during the COVID-19 pandemic: early results 
from New York. J Orthop Trauma 34(8):403-410. https://doi.org/ 10.1097/BOT.0000000000001849

5. Wang KC, Xiao R, Cheung ZB, Barbera JP, Forsh DA (2020) Early mortality after hip fracture surgery in COVID-19 patients: a systematic review and meta-analysis. J Orthop 22:584-591. https://doi.org/10.1016/j.jor.2020.11.012

6. Chen N, Zhou M, Dong X et al (2020) Epidemiological and clinical characteristics of 99 cases of 2019 novel coronavirus pneumonia in Wuhan, China: a descriptive study. Lancet 395(10223):507513. https://doi.org/10.1016/S0140-6736(20)30211-7

7. Ryoo SM, Han KS, Ahn S et al (2019) Korean Shock Society (KoSS) Investigators. The usefulness of C-reactive protein and procalcitonin to predict prognosis in septic shock patients: A multicenter prospective registry-based observational study. Sci Rep 9(1):6579. https://doi.org/10.1038/s41598-019-42972-7

8. Wang D, Li R, Wang J et al (2020) Correlation analysis between disease severity and clinical and biochemical characteristics of 143 cases of COVID-19 in Wuhan, China: a descriptive study. BMC Infect Dis 20(1):519. https://doi.org/10.1186/ s12879-020-05242-w

9. Yang R, Chang Q, Meng X, Gao N, Wang W (2018) Prognostic value of Systemic immune-inflammation index in cancer: a metaanalysis. J Cancer 9(18):3295-3302. https://doi.org/10.7150/jca. 25691

10. Huang H, Liu Q, Zhu L et al (2019) Prognostic value of preoperative systemic immune-inflammation index in patients with cervical cancer. Sci Rep 9(1):3284. https://doi.org/10.1038/ s41598-019-39150-0

11. Ministry of Health of Turkey, Public Health Directorate (2020). COVID-19 - new coronavirus disease. Available at: https://covid 19bilgi.saglik.gov.tr/tr. Accessed 2 Jun 2020.

12. Ministry of Health of Turkey, Public Health Directorate (2019). National pandemic influenza preparedness plan. Available at: https://hsgm.saglik.gov.tr/tr/bulasicihastaliklar-haberler/ulusalpandemi-hazirlik-plani.html. Accessed 25 May 2020.

13. Ministry of Health of Turkey, Public Health Directorate (2020). COVID-19 treatment protocol- version 1. Available at: https:// covid19bilgi.saglik.gov.tr/ tr/algoritmalar. Accessed 30 Jan 2020.

14. Egol KA, Konda SR, Bird ML, et al; NYU COVID Hip Fracture Research Group. (2020) Increased mortality and major complications in hip fracture care during the COVID-19 pandemic: a New York City perspective. J Orthop Trauma. 34(8):395-402 https:// doi.org/10.1097/BOT.0000000000001845

15. Muñoz Vives JM, Jornet-Gibert M, Cámara-Cabrera J, et al; Spanish HIP-COVID Investigation Group. (2020) Mortality rates of patients with proximal femoral fracture in a worldwide pandemic: preliminary results of the Spanish HIP-COVID observational study. J Bone Joint Surg Am. 102(13):e69 https://doi.org/10.2106/ JBJS. 20.00686

16. Al Farii H, Al Rawahi S, Samaila E, Lavini F, Magnan B, Al MS (2020) Thirty-day mortality in COVID-19 positive patients with hip fractures: a case-series and literature review. Geriatr Orthop Surg Rehabil 11:2151459320972681. https://doi.org/10.1177/ 2151459320972681

17. Roberts SE, Goldacre MJ (2003) Time trends and demography of mortality after fractured neck of femur in an English population, 1968-98: database study. BMJ 327(7418):771-775. https://doi. org/10.1136/bmj.327.7418.771

18. Wignall A, Giannoudis V, De C et al (2021) The impact of COVID-19 on the management and outcomes of patients with proximal femoral fractures: a multi-centre study of 580 patients. J Orthop Surg Res 16(1):155. https://doi.org/10.1186/ s13018-021-02301-Z

19. Kumar P, Jindal K, Aggarwal S, Kumar V, Rajnish RK (2021) 30-day mortality rate in hip fractures among the elderly with coexistent COVID-19 infection: a systematic review. Indian J Orthop 55(3):1-11. https://doi.org/10.1007/s43465-021-00386-6

20. Kayani B, Onochie E, Patil V et al (2020) The effects of COVID19 on perioperative morbidity and mortality in patients with hip fractures. Bone Joint J 102-B(9):1136-1145. https://doi.org/10. 1302/0301-620X.102B9.BJJ-2020-1127.R1

21. Huang I, Pranata R, Lim MA, Oehadian A, Alisjahbana B (2020) $\mathrm{C}$-reactive protein, procalcitonin, D-dimer, and ferritin in severe coronavirus disease-2019: a meta-analysis. Ther Adv Respir Dis 14:1753466620937175. https://doi.org/10.1177/1753466620 937175

22. Liu F, Li L, Xu M et al (2020) Prognostic value of interleukin-6, C-reactive protein, and procalcitonin in patients with COVID-19. J Clin Virol 127:104370. https://doi.org/10.1016/j.jcv.2020.104370

23. Ruan Q, Yang K, Wang W, Jiang L, Song J (2020) Clinical predictors of mortality due to COVID-19 based on an analysis of data of 150 patients from Wuhan. China Intensive Care Med 46(5):846848. https://doi.org/10.1007/s00134-020-05991-x

24. Sedlář M, Kvasnička J, Krška Z, Tománková T, Linhart A (2015) Early and subacute inflammatory response and long-term survival after hip trauma and surgery. Arch Gerontol Geriatr 60(3):431436. https://doi.org/10.1016/j.archger.2015.02.002

Publisher's note Springer Nature remains neutral with regard to jurisdictional claims in published maps and institutional affiliations. 